\title{
The Sun, the Earth's Atmosphere, and Radio Transmission.
}

\section{By Prof. S. Chapman, F.R.S.}

$\mathrm{T}$ HE immense present-day importance of radio communication has directed the attention of a wider circle than ever before to the conditions prevailing at great heights in the atmosphere. Even to the professional meteorologist it seemed formerly that what happens above a height of 30 or 40 kilometres has no bearing on the practical affairs of humanity, however interesting the problems of these upper levels might be to the pure theorist. Almost the only influence of these levels upon the technical operations of mankind seemed to consist in the occasional interruption of telegraphic communication at times of great magnetic storms and auroral displays ; the interference was pretty clearly due to currents induced in the earth and the cables by the rapidly varying magnetic field, but the direct association of the latter with the upper atmosphere was somewhat hypothetical, though the auroræ were quite indubitably atmospheric phenomena.

Now that signal transmission is not confined to cables, and electromagnetic oscillations over a wide range of wave-length are sent out into space, the upper air assumes more direct importance, for it is known that a large part of the energy transmitted to distant points travels intermediately at high levels. The manner in which the waves are affected in the upper levels is already partly understood; the rate of propagation is increased by the presence of ions, so that if the ionisation increases with height the upper part of a wave front travelling at an upward inclination to the horizontal is tilted forward, and the wave train may thus be refracted downwards again, quite sharp angles of bending being possible. At the same time, some of the energy of the wave, communicated to the ions, is lost by the latter during collisions with surrounding molecules ; if the mean free path of the ions is short, and collisions frequent, the energy of the waves may be rapidly absorbed; the absorption increases with the density of the ionised layer, and is greater for long (low frequency) waves than for short waves. Moreover, the earth's magnetic field deflects the ions while they are oscillating under the influence of the waves, and brings about a change of polarisation of the waves, which is responsible for some of the errors in direction-finding by wireless.

These facts make it clear that changes in the ionisation of the upper atmosphere have an important bearing on wireless transmission, and should be carefully investigated in order that, if possible, the methods of signalling may be adjusted to the changing conditions. Probably in the future the upper atmospheric phenomena will become known by wireless investigations themselves with a thoroughness and detail not otherwise attainable - the results already achieved seem to promise this. But, at least in these early days of such work, some help towards the understanding of wireless problems may be gained from the knowledge slowly accumulated during three or four generations by the study of solar phenomena, auroræ, and terrestrial magnetism.

The ionisation of the atmosphere at high levels may be partly due to the penetrating radiation studied by Kolhörster, Hess, and Millikan, or by the runaway electrons the probable occurrence of which during thunderstorms has been pointed out by C. T. R. Wilson. It seems unlikely, however, that the ionisation thus produced is comparable in importance with that due to solar action. The magnetic variations are intimately connected with the ionisation of the upper atmosphere, and indicate its general distribution and its more important changes, which are unmistakably associated with the state of the sun, or its presentation towards the earth. Further study may reveal magnetic phenomena indicative of ionisation unconnected with the sun, but it appears improbable that such can be of the same order of magnitude as that of which the sun is obviously the cause.

The sun ionises the upper air by two independent agents. One of these travels rectilinearly and falls only on the hemisphere of the earth which faces the sun, that is, on the day or sunlit hemisphere. Many facts indicate that this agent is ultra-violet radiation, absorbed in the upper atmosphere, principally by ozone, itself produced by the first stage of such absorption; another consequence is that the temperature of the layer in question (extending upwards from about $50 \mathrm{~km}$. height) is raised above that of the air at ground-level. As the revolving earth carries any particular region of the atmosphere into the night, when the ionising radiation is cut off, the ions re-combine, and most rapidly at the lower surface, which in the course of a few hours moves upward from a level of about $50 \mathrm{~km}$. to $100 \mathrm{~km}$. or more. The conductivity of the ionised layer is consequently diminished at night, by the reduction in both the thickness and the specific conductivity; this affects the electric currents flowing in the ionised layer, to which the daily magnetic variations are due. The latter are much more intense by day than by night, and in low latitudes (where the sun's rays are most direct) than in regions farther from the equator. The magnetic variations also show clearly that the conductivity of the layer ionised in this way is distinctly greater (by at least 25 per cent.) at sunspot maximum than at sunspot minimum; this requires a change of intensity in the ultraviolet radiation by about 60 per cent., which is remarkable in view of the absence of any comparable variation, during the sunspot cycle, in the sun's visible radiation. The increase in the ultraviolet radiation seems to be due to enhanced emission from the sun's surface as a whole, and to be independent of particular disturbed localities on the sun.

The other solar ionising agent is corpuscular, and proceeds from locally disturbed areas on the sun's surface, often, though not always, associated with visible markings such as sunspots. The corpuscles

No. 2994, VoL. 119] 
are ejected in laterally limited streams, the direction of which varies as the sun rotates. When such a stream happens to impinge on the earth, the air becomes highly ionised in the atmospheric regions affected, which are situated geographically within about $20^{\circ}$ of the poles of the axis of magnetisation of the earth, though they spread farther towards the equator when the streams are specially intense. The corpuscles can penetrate the earth's atmosphere down to not less than $80 \mathrm{~km}$. above the ground; they impinge not only on the side of the earth facing the sun, but also bend round to the right side of the earth, where they produce visible auroræ. The deflexion of the corpuscles towards the magnetic poles is due to the earth's magnetic field, and indicates that the streams have a slight excess charge and are ionised.

The ionisation of the auroral zones renders these regions of the atmosphere highly conducting, and permits intense electric currents to flow in them; the changing magnetic field of these currents is observed as a magnetic storm (or lesser disturbance). Even if auroræ were not visible, the magnetic observations would indicate the existence of highly conducting upper air in polar regions.

The ionisation of the air due to the solar corpuscles is much less uniformly distributed than the ionisation over the sunlit hemisphere due to ultra-violet radiation; the limited distribution of bright auroræ gives visible indication of this. Also the ionisation by corpuscles varies irregularly with time, and cannot be predicted at present with any certainty ; it depends both on the activity and presentation (towards the earth) of the disturbed solar areas. If such an area remains active after the lapse of a solar rotation period (about 27 days), it may affect the earth a second time, or even several times after successive rotations. Hence arises the clearly marked tendency for magnetic disturbance to recur after 27 days; but this is only a tendency, for the direction of the stream from a solar disturbed area may change, or the area may not remain long active.

The influence upon wireless transmission of the regular change of ionisation from day to night has long been recognised; the improved conditions at night, especially for long waves, are naturally accounted for by the withdrawal of the refracting ionised layer to greater heights at night, where the ions have longer free paths and where their less frequent collisions dissipate less of the energy acquired from the electric waves. The influence upon wireless of the less regularly distributed but occasionally very intense ionisation due to solar corpuscles has only Jately become reasonably certain. The effects are likely to be complicated, and it will doubtless be a long time before they are clearly ascertained. An interesting feature suggested by recent observations is that transmission from Europe to America is more affected than that from Europe to the East. This is not unexpected, in view of the inclination of the earth's magnetic to its geographical axis; the centre of the north auroral zone is about $10^{\circ}$ from the geographical north pole, in the direction of Canada, and auroræ are observed in Canada in much lower latitudes than in Europe. The region of abnormal corpuscular ionisation will therefore extend about $20^{\circ}$ farther towards the equator in Canada than in Russia.

\section{The Decimal Classification of Melvil Dewey and its Extension by the Brussels Institute of Bibliography.}

By Dr. S. C. Bradford, The Science Library, S.W.7.

$\mathrm{T}^{\mathrm{H}}$ HE study of classification is a necessary preliminary to the preparation of a comprehensive guide to recorded information. Of the multifarious systems that have been suggested for the classification of literature, Dewey's "Decimal Classification and Relative Index," first developed in 1873 , seemed the most hopeful of any produced hitherto, and was hailed with jubilation in many quarters. But, although it proved successful for the purpose for which, in the first place, it was designed, i.e. the classification of books, it has been found inadequate when applied to greater bibliographical detail, such as individual scientific and technica] papers. In its original form it is therefore unsuitable for the preparation of a detailed index to the records of human work and thought.

Dewey's scheme comprises two parts : a classification in which more or less logically arranged subdivisions of knowledge are given separate arbitrary numbers, and an alphabetical index of subjects, regarded by Dewey as the more important feature of the system, to indicate the respective numbers of the subdivisions. The index aims at giving similar or synonymous words and the same words in different connexions, so that any person of intelligence can scarcely fail to get the right number. In this scheme the whole of knowledge is represented by unity, and divided into ten main divisions. The first, comprising all numbers between 0.0 and 0.1 , is assigned to works dealing with general subjects such as encyclopædias, bibliographies, etc., and those that cannot be allocated to the nine more definite subdivisions that follow. These are :
$0 \cdot 1$ Philosophy.
0.2 Religion.
0.6 Applied Science.
0.7 Fine Arts.

0.3 Social Sciences, Law. 0.8 Literature.

0.4 Philology, Languages, 0.9 History, Geography, 0.5 Pure Science. Biography.

Each of these main groups is again subdivided by addition of another decimal place, and further subdivision of these secondary and succeeding groups is obtained as desired. Thus :

0.5 Pure Science. 0.5315 Gravity, Ballistics. 0.53 Physics. 0.531 Mechanics. the Energy of Projectiles.

In practice the initial decimal point is omitted, and 